\title{
Modulation of microRNA processing by mismatch repair protein MutLa
}

\author{
Guogen $\mathrm{Mao}^{1}$, Sanghee $\mathrm{Lee}^{2}$, Janice Ortega ${ }^{1}$, Liya Gu${ }^{1,3}$, Guo-Min $\mathrm{Li}^{1,2,3}$ \\ ${ }^{I}$ Graduate Center for Toxicology, University of Kentucky College of Medicine, Lexington, KY 40536, USA, ${ }^{2}$ Graduate Center for \\ Nutritional Sciences, University of Kentucky College of Medicine, Lexington, KY 40536, USA, ${ }^{3}$ Markey Cancer Center, University \\ of Kentucky College of Medicine, Lexington, KY 40536, USA
}

MicroRNAs (miRNAs) are critical post-transcriptional regulators and are derived from hairpin-shaped primary transcripts via a series of processing steps. However, how the production of individual miRNAs is regulated remains largely unknown. Similarly, loss or overexpression of the key mismatch repair protein MutLa (MLH1-PMS2 heterodimer) leads to genome instability and tumorigenesis, but the mechanisms controlling MutL $\alpha$ expression are unknown. Here we demonstrate in vitro and in vivo that MLH1 and miR-422a participate in a feedback loop that regulates the level of both molecules. Using a defined in-vitro miRNA processing system, we show that MutLa stimulates the conversion of pri-miR-422a to pre-miR-422a, as well as the processing of other miRNAs tested, implicating MutL $\alpha$ as a general stimulating factor for miRNA biogenesis. This newly identified MutLa function requires its ATPase and pri-miRNA binding activities. In contrast, miR-422a downregulates MutL $\alpha$ levels by suppressing MLH1 expression through base pairing with the $M L H 1$ 3'-untranslated region. A model depicting this feedback mechanism is discussed.

Keywords: mismatch repair; MLH1; MutL $\alpha$; miRNA; Drosha; DGCR8; 3'-UTR

Cell Research (2012) 22:973-985. doi:10.1038/cr.2012.18; published online 31 January 2012

\section{Introduction}

MicroRNAs (miRNAs) are short ( 22s nucleotide) single-stranded RNAs that function as gene expression regulators by binding to and destabilizing or inhibiting translation of target mRNAs [1-3]. In mammalian cells, primary miRNA transcripts (pri-miRNAs) are initially processed in the nucleus into the $\sim 70$-nt hairpin RNAs termed precursor miRNAs (pre-miRNAs), which are further processed in the cytoplasm to yield mature miRNAs [1-3]. The processing of pri-miRNAs to pre-miRNAs requires the RNase III Drosha and its partner DGCR8, with the former functioning as the catalytic subunit and the latter recognizing the RNA substrate [4-7]. Although recent studies have implicated other factors in miRNA biogenesis, which include p53 [8], Smad [9, 10] and ATM [11], the molecular basis by which miRNA biogenesis is

Correspondence: Liya Gu

E-mail:1gu0@uky.edu

Received 24 August 2011; revised 30 November 2011; accepted 13 December 2011; published online 31 January 2012 regulated is not fully understood.

miRNAs have been shown to regulate diverse cellular processes, including the DNA mismatch repair (MMR) pathway [12-14], a major genome maintenance system. The importance of MMR in genome maintenance is demonstrated by the fact that defects in the system cause genome instability and susceptibility to human cancers [15-19]. The MMR functions that protect and maintain the integrity of the genome in mammalian cells include correcting DNA biosynthetic errors, suppressing homeologous recombination and mediating DNA damage signaling [20-23]. It is well established that MMR initiation factors, such as mammalian MutS and MutL homolog proteins, are essential in all MMRmediated cellular processes. There are at least three functional MutL homolog proteins in human cells, which are MutL $\alpha$ (MLH1-PMS2), MutL $\beta$ (MLH1-PMS1) and MutL $\gamma$ (MLH1-MLH3). Although the roles of MutL $\beta$ and MutL $\gamma$ in MMR-associated genome maintenance remain to be characterized, the role of MutL $\alpha$ in these functions is well established [23]. Because MLH1 is the obligating subunit in all these heterodimers, its cellular 
level is critical to the individual complexes and MMRdependent genome-maintenance functions. Whereas no or low MLH1 results in genome instability and predisposition to cancer $[16,17,19]$, overexpression of MLH1 induces apoptosis [24] and/or a mutator phenotype [25, 26]. Therefore, precise regulation of the cellular level of MLH1 is critical for genome stability. Although miRNAs likely play an important role in regulating MLH1 expression [12-14], the mechanism of the regulation reaction is unclear. Interestingly, recent studies suggest that MLH1 may modulate miRNA processing, as MLH1 expression determines miRNA expression patterns in colorectal tumors $[27,28]$. These observations suggest a complex regulation relationship between MLH1 and miRNAs.

In this study, we demonstrate that miRNAs and MLH1 participate in a regulatory feedback loop, in which miRNA-422a negatively regulates the expression of MLH1 protein and the MLH1-PMS2 heterodimer (MutL $\alpha$ ) positively regulates the processing of miR-422a and other miRNAs. In-vitro studies reveal that MutL $\alpha$ specifically binds to pri-miRNAs, interacts with the Microprocessor complex Drosha/DGCR8, and stimulates the Drosha/ DGCR8-catalyzed processing of pri-miRNAs to premiRNAs in a manner dependent on MutL $\alpha$ ATPase and pri-miRNA-binding activities. These observations reveal a novel MutL $\alpha$ function in regulating miRNA biogenesis and a novel feedback mechanism that precisely regulates the cellular level of MutL $\alpha$ via miRNA functions.

\section{Results}

miR-422a regulates $M L H 1$ expression by interacting with the MLH1 3'-UTR

Our previous studies showed that a three-nucleotide deletion in the MLH1 $3^{\prime}$-UTR is associated with leukemia relapse $[12,29]$. Computational analysis using MicroInspector [30] identified a putative binding site for miR-422a in the MLH1 3'-UTR that is disrupted by the 3-nucleotide deletion, suggesting a possible role for miR422a in regulating expression of MLH1. To explore this possibility, HeLa cells were transfected with miR-422a precursor (pre-miR-422a) or anti-miR-422a, and the MLH1 protein level was quantified. The results showed that transfection of pre-miR-422a significantly lowered the level of MLH1 $(P<0.001)$, while transfection of anti-miR-422a increased the level of MLH1 (Figure 1A and 1B). The level of MLH1 mRNA was affected in a similar manner (Figure 1C). These results are consistent with the hypothesis that miR-422a negatively regulates MLH1 expression in HeLa cells.

To determine if the repression of MLH1 expression is miR-422a-specific, the MLH1 protein levels were mea- sured in HeLa cells transfected with pre-/anti-miR-16-1 or pre-/anti-miR-30a. The results show that the MLH1 level is essentially not interfered by either miR-16-1 or miR-30a (Figure 1D and 1E). These experiments ruled out the potential side effect of miR-422a transfection that might affect the MLH1 protein level. However, it is worth mentioning that HeLa cells transfected with 25 pmol of anti-miR-422a had an MLH1 protein level that is lower than that in untransfected cells (Figure 1A, compare lanes 5 and 6). This reduction in MLH1 expression is likely caused at the transcriptional level, but not at the translational level, as judged by the fact that (1) the lower dose anti-miRNA-transfected cells expressed almost the lowest level of MLH1 mRNA (Figure 1C), and (2) despite that, the cells transfected with 25 pmol of anti-miRNA and those transfected with 200 pmol of premiRNA expressed a comparable level of MLH1 mRNA (Figure 1C), the former cells exhibited an MLH1 protein level almost as twice as that in the latter cells (Figure 1B). However, how a low level of anti-miR-422a negatively influences the $M L H 1$ transcription remains to be investigated.

To determine if miR-422a regulates $M L H 1$ expression by binding to a putative miR-422a response element (RE) in the +18 to +50 region of the MLH1 3'-UTR, three tandem copies of the putative miR-422a RE $(3 \times$ miR422aRE) were cloned into the pMIR-MLH1 vector to generate pMIR-MLH1-3×miR422aRE (Figure 1F). The resulting plasmid was co-transfected with pre- or anti-miR-422a into $M L H 1$-defective 293T cells [31]. Western blotting analysis showed that the level of MLH1 expression in 293 T cells (Figure $1 \mathrm{G}$ and $1 \mathrm{H}$ ) was down- or upregulated in a dose-dependent manner by pre- or anti-miR422a, respectively. Comparable effects on ectopic luciferase expression were also observed when pMIR-Luc$3 \times$ miR422aRE was co-expressed with pre- or anti-miR422a in 293T and HEK 293 cells (data not shown). These results are consistent with the notion that miR-422a represses MLH1 expression by binding to the region of +18 to +50 in the MLH1 $3^{\prime}$-UTR.

\section{Correlation between expression of MLH1 protein and pre-miR-422a}

The suppression of $M L H 1$ expression by miR-422a prompted us to think that a high level of endogenous miR-422a may have contributed to the MMR defect in $293 \mathrm{~T}$ cells, which has previously been attributed to hypermethylation of the $M L H 1$ promoter [31]. To test this possibility, we performed quantitative RT-PCR to determine the levels of pre- and pri-miR-422a in $293 \mathrm{~T}$ cells using RNAs isolated from cytoplasm and nuclei, respectively. As shown in Figure 2B, pri-miRNAs appear 


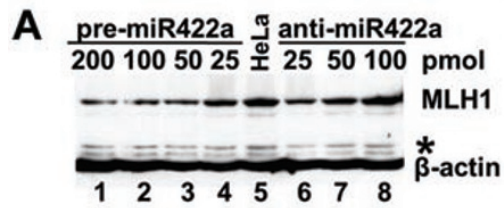

B

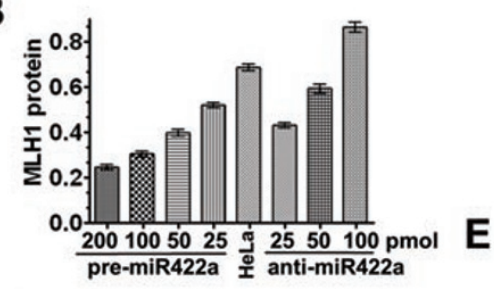

\section{C}

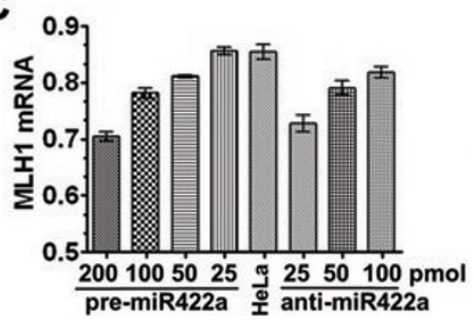

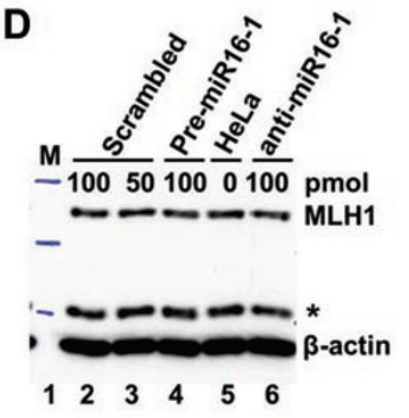

\section{E}

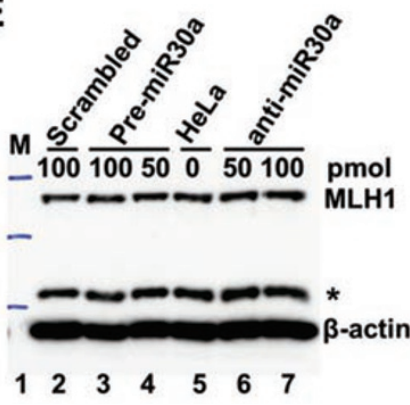

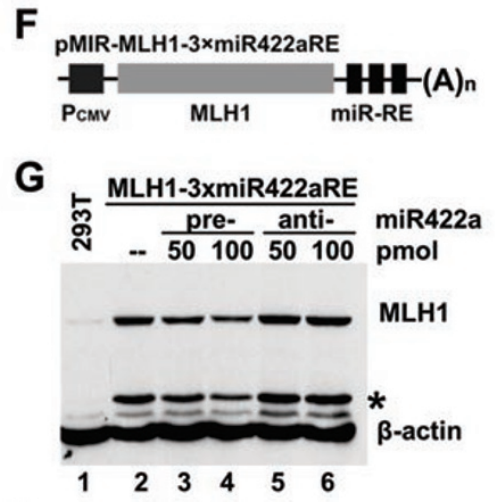

H

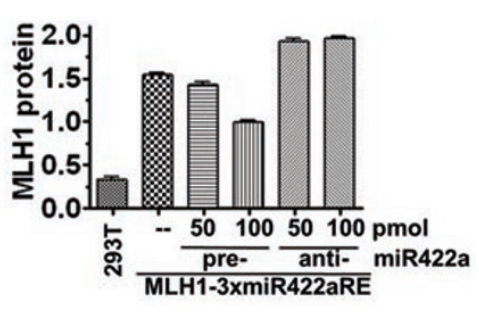

Figure 1 miR-422a regulates MLH1 expression via interaction with MLH1 3'-UTR. (A-C) Effect of miR-422a on endogenous MLH1 expression. Pre-miR-422a or anti-miR-422a was transfected into HeLa cells as indicated, and the endogenous MLH1 protein (A, B) and mRNA (C) were measured by western blotting or qRT-PCR, respectively. (D, E) Effect of miR-16-1 and miR-30a on exogenous MLH1 expression. The experiments were performed similarly as described in (A), but with pre-/ anti-miR-16-1 and pre-/anti-miR-30a, as indicated. (F) Expression construct containing MLH1 and three tandem copies of the putative miR-422aRE (3×miR422aRE) in the 3'-UTR (G, H) Effect of miR-422a on exogenous MLH1 expression. pMIRMLH1-3×miR-422aRE was co-transfected with pre-miR-422a or anti-miR-422a into MLH1-deficient 293T cells, and MLH1 protein was determined by western blotting and normalized against the amount of MLH1 protein in cells without miR-422a transfection. Data represent three independent experiments (mean \pm SD). Statistical significance was determined by one-way ANOVA. *Degraded MLH1.

to predominantly localize in nuclei, while pre-miRNAs are mainly present in cytoplasm, indicating translocation of pre-miRNAs from the nucleus to cytoplasm upon their production [32]. Surprisingly, despite that the level of pri-miR-422 in both $293 \mathrm{~T}$ and 293 cells is comparable, the level of pre-miR-422a in MLH1-deficient 293T cells is significantly lower than in $M L H 1$-proficient 293 cells (Figure 2B). It was noted that the amount of pre-miR422a amplified from nuclear RNAs of 293T and 293 cells was essentially the same (Figure 2B). We believe that these products were derived mostly from the primiR-422a template in the nucleus, as the primers for premiR-422a could also amplify the pre-miRNA portion of the pri-miRNA (see Figure 2A). Similar results were also obtained with $M L H 1$-deficient and $M L H 1$-proficient leukemia patient cells, i.e., MLH1-deficient AML0606 cells express significantly less pre-miR-422a than their isogenic $M L H 1$-proficient AML0805 cells $(P<0.01$; Figure 2C, lanes 3 and 4$)$. These results strongly suggest that MLH1 positively regulates miR-422a processing from pri-miR-422a to pre-miR422a.

To test this hypothesis, we first knocked down MLH1 expression by siRNA in HeLa cells. As expected, the knockdown led to significant MLH1 reduction at both protein (Figure 2D) and mRNA levels (Figure 2E, left panel). We then quantified the pre- and pri-miR422a levels by qRT-PCR in the MLH1-knockdown cells. The results show that the level of pre-miR-422a (Figure $2 \mathrm{E}$, middle), but not that of pri-miR-422a (Figure 2E, right), decreased significantly in the knockdown cells $(P$ $<0.01)$. We further analyzed the levels of pri- and premiR-422a in MLH1-deficient 293T cells transfected with a plasmid (pcDNA3.1) carrying either a wild-type (WT) MLH1 gene or an MLH1 gene whose translational start codon (ATG) was changed to a stop codon (Figure 3A). As expected, the ectopic MLH1 expression was only observed in 293T cells transfected with WT MLH1 but not the mutant MLH1 (Figure 3B). Conversely, level of increased pre-miR-422a (Figure 3C, ANOVA, $P<0.001$ ), but not pri-miR-422a (Figure 3D), was detected in cells 
transfected with WT MLH1. Similar results were also obtained in MLH1-deficient HCT116 cells transfected with these plasmids (data not shown). These observations indicate that higher ectopic MLH1 expression correlates with higher endogenous pre-miR-422a level, supporting the notion that MLH1 expression promotes the production of pre-miR-422a.

\section{MutL $\alpha$ interacts with pri-miRNA and Microprocessor}

The conversion of pri-miRNA to pre-miRNA is cata- lyzed by the Drosha/DGCR8 complex [1-3], also known as Microprocessor. Because MutL $\alpha$ (MLH1-PMS2 heterodimer) is the most important and abundant protein among MLH1-formed MutL heterodimers [23], MutL $\alpha$ was analyzed for its potential role to stimulate pri-miR422a processing by Microprocessor. We first tested if purified MutL $\alpha$ enhances the interaction between purified Microprocessor (Figure 4A) and pri-miR-422a, using electrophoretic mobility shift assays (EMSAs). In the absence of MutL $\alpha$, efficient shift of ${ }^{32} \mathrm{P}$-labeled pri-miR-
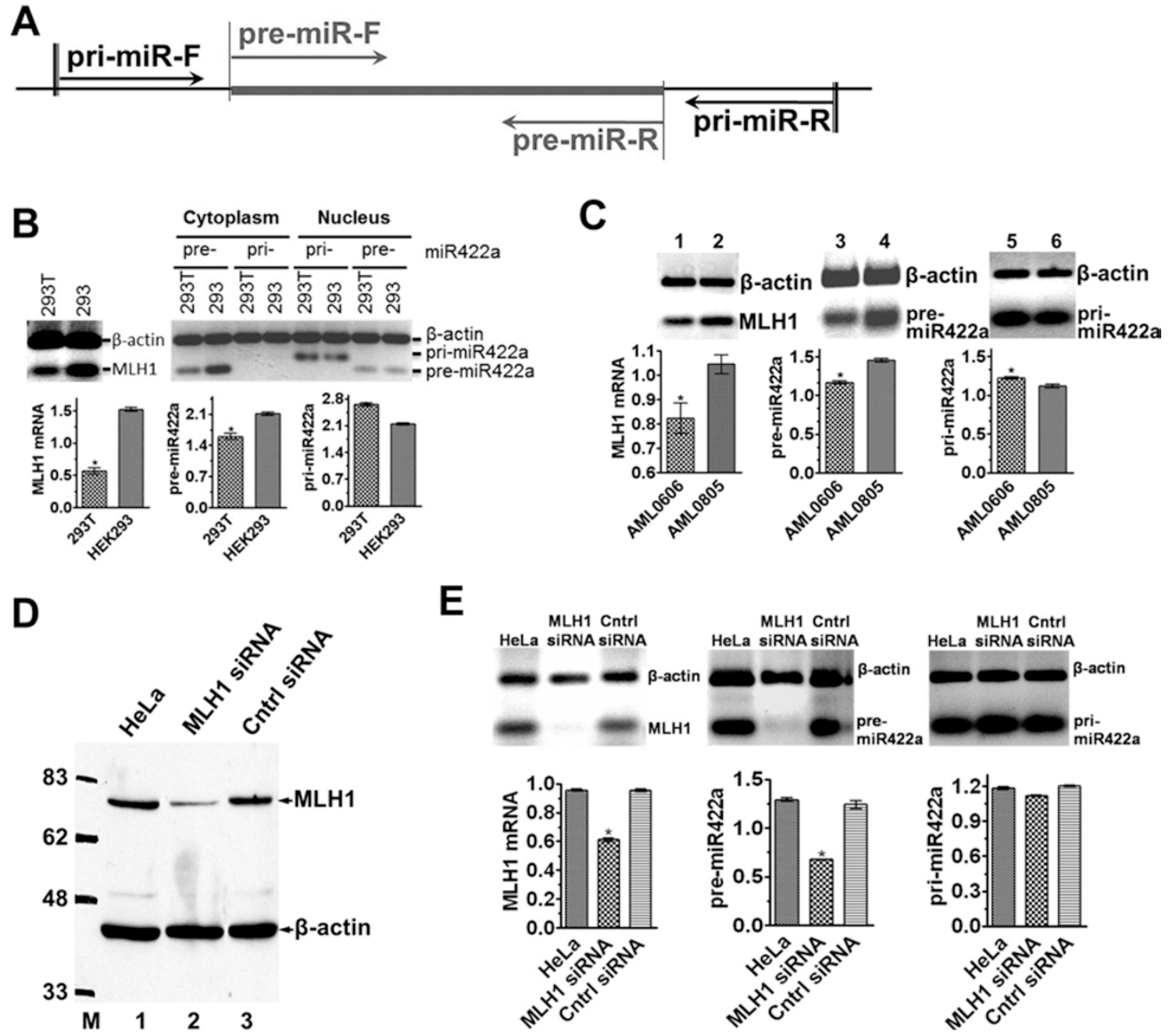

Figure 2 Correlation between levels of MLH1 protein and pre-miR-422a. (A) Schematic diagram of primers used to quantify pre-miR-422a and pri-miR-422a. Primers for pri-miR422a amplify a 198-bp fragment, but primers for pre-miR422a-amplify an 83-bp fragment. (B) Endogenous expression of MLH1 mRNA, pre- and pri-miR422a in 293T and 293 cells. (C) Expression of MLH1 mRNA, and pre- and pri-miR422a in an AML patient before and after relapse. (D) MLH1 knockdown in HeLa cells by anti-MLH1-siRNA. (E) Effect of MLH1 knockdown on expression of pre- and pri-miR-422a in HeLa cells. The levels of preand pri-miR-422a were determined by qRT-PCR using RNAs isolated from cytoplasm and nuclei as templates, respectively. PCR products from $\beta$-actin mRNA were used as internal controls. Data represent three independent experiments (mean \pm SD). Statistical significance was determined by either Student's $t$-test (C) or one-way ANOVA (E). MLH1 protein was determined by western blotting. 

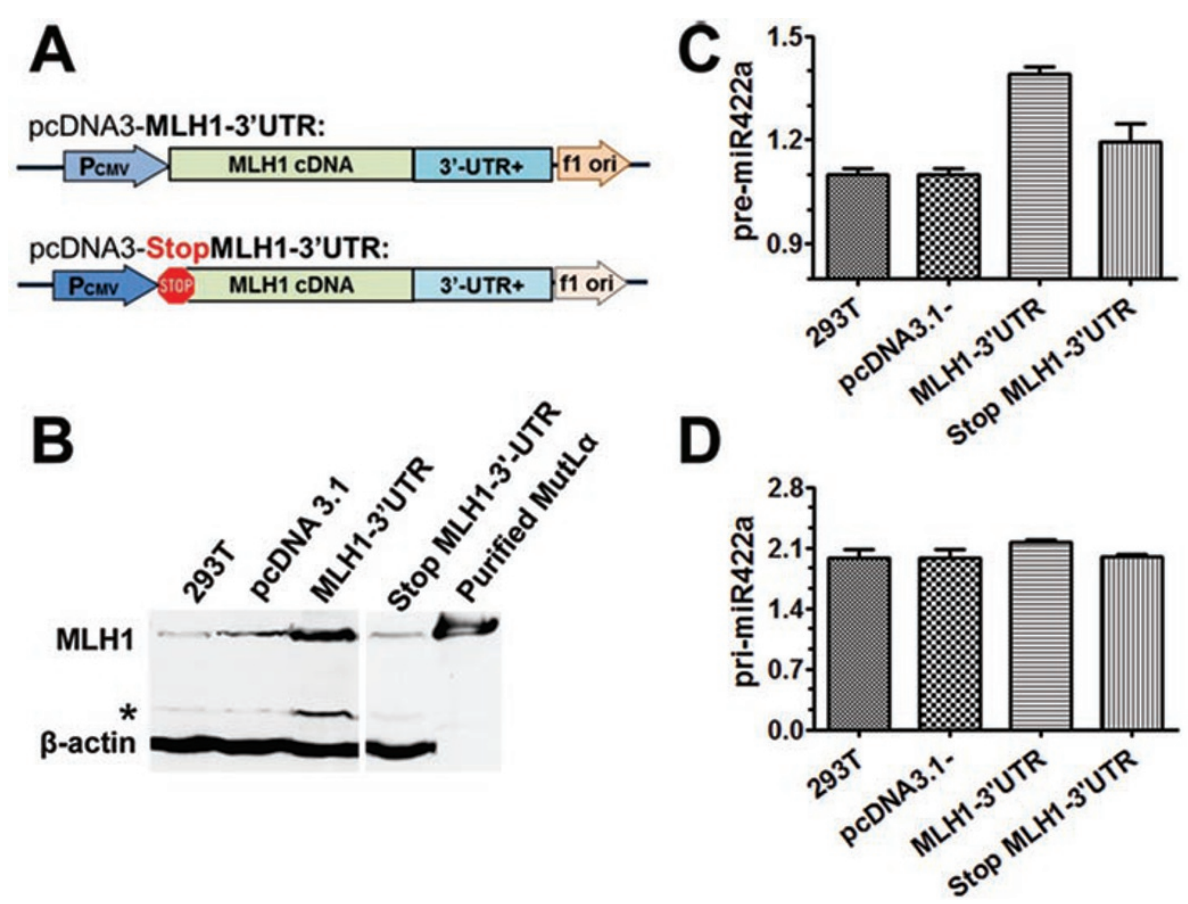

Figure 3 Expression of ectopic MLH1 protein increases the level of pre-miR-422a in 293T cells. (A) Plasmid pcDNA3.1 constructs carrying WT MLH1 cDNA and a mutant MLH1 gene whose ATG start codon was changed to a TAA stop codon by site-directed mutagenesis. (B) MLH1 expression in MLH1-deficient 293T cells transfected with pcDNA3.1 alone, pcDNA3.1 carrying WT or mutant $M L H 1$, as indicated. Ectopic MLH1 protein was detected by western blotting, with $\beta$-actin serving as an internal loading control. (C, D) qPCR quantification of pre-miR-422a (C) and pri-miR-422a (D) in 293T cells transfected with the indicated plasmids. Data shown are from three independent experiments (mean $\pm \mathrm{SD}$ ). Statistical significance was determined by one-way ANOVA. *Degraded MLH1.

422a required a DGCR8 concentration of $1.5 \mu \mathrm{M}$ (Figure $4 \mathrm{~B}$, lane 3). However, addition of $0.15 \mu \mathrm{M}$ MutL $\alpha$ to the reaction reduced the required DGCR8 concentration for pri-miRNA binding to $0.5 \mu \mathrm{M}$ (Figure 4B, lane 7), suggesting that MutL $\alpha$ enhances DGCR8 interaction with pri-miR-422a. Interestingly, a band that is different from the DGCR8-pri-miR-422a complexes appeared in the reaction containing MutL $\alpha$, but no DGCR8 (Figure 4B, lane 5), indicating binding of the pri-miRNA by MutL $\alpha$. Additionally, when DGCR8 concentration increased, more slowly migrated complexes were evident (see arrows in Figure 4B, lanes 7-9), suggesting that MutL $\alpha$ induces supershifts, which could be due to MutL $\alpha$ interactions with the pri-miRNA and/or DGCR8-pri-miRNA complex. Similar results were also observed when MutL $\alpha$ was added to the Drosha-pri-miR-422a complex (Figure 4C). Taken together, these observations suggest that MutL $\alpha$ interacts with both pri-miR-422a and the Microprocessor proteins.

EMSAs were performed to determine direct binding of MutL $\alpha$ to pri-miR-422a. As shown in Figure 4D, MutL $\alpha$ indeed specifically shifted ${ }^{32} \mathrm{P}$-labeled pri-miR-422a even in the presence of excess, unlabeled competitor tRNA. Pri-miR-422a-binding reactions were also carried out with several MMR-deficient MutL $\alpha$ mutants, MutL $\alpha E A$ $\left(\mathrm{MLH1}_{\mathrm{E} 34 \mathrm{~A}}-\mathrm{PMS} 2_{\mathrm{E} 41 \mathrm{~A}}\right)[33,34]$, MutL $\alpha \mathrm{F} 99 \mathrm{~L}\left(\mathrm{MLH1}_{\mathrm{F} 99 \mathrm{~L}}-\right.$ PMS2) [29] and MutL $\alpha$ E705K (MLH1-PMS2 ${ }_{\text {E705K }}$ ) [35]. While MutL $\alpha$ EA (Figure 4D, lanes 6-7) and MutL $\alpha$ F99L (Figure 4D, lanes 8-9) bound pri-miR-422a as efficiently as the WT MutL $\alpha$, MutL $\alpha$ E705K failed to interact with pri-miR-422a (Figure 4D, lanes 10-11). These results demonstrate that MutL $\alpha$ has significant in-vitro affinity for pri-miR-422a, which may play an important role in regulating miRNA processing.

Co-immunoprecipitation experiments were performed to determine MutL $\alpha$ interactions with Drosha and DGCR8 in HeLa extracts. The result revealed that an MLH1 antibody could pull down not only MLH1, but also Drosha and DGCR8 (Figure 4E, lane 4). This pulldown was not due to the binding of the individual proteins to endogenous pri-miRNAs, as RNase A-treated coimmunoprecipitation gave the same outcome (compare lanes 4 and 5). Similarly, the MLH1 antibody could pull down Drosha and DGCR8, as well as PMS2, when all 
purified proteins were used in the co-immunoprecipitation experiment (Figure 4F). These results suggest that MutL $\alpha$ may form a complex with Microprocessor.

MutLa stimulates pri-miRNA processing in vitro and in vivo

Based on the fact that MutL $\alpha$ interacts with both the substrate and enzymes of miRNA processing, we hypothesize that the ability for MLH1 to facilitate miR-422a production in cells is likely due to direct participation of MutL $\alpha$ in miRNA biogenesis. This hypothesis was first tested using an in-vitro assay. For this purpose, we established conditions that support miRNA processing in vitro, and demonstrated that Drosha and DGCR8 efficiently convert ${ }^{32} \mathrm{P}$-labeled pri-miRNAs to pre-miRNAs (Figure 5). To our knowledge, a successful in-vitro reconstitution of miRNA processing using purified proteins has not been reported. In this reconstituted miRNA-processing assay, we incubated ${ }^{32} \mathrm{P}$-labeled pri-miRNA with recombinant Drosha and DGCR8 proteins in the presence or absence of MutL $\alpha$. As shown in Figure 5A, addition of purified MutL $\alpha$ into the reconstituted reactions stimulated conversion of pri-miR-422a to pre-miR-422a by Drosha and DGCR8 (compare lanes 1-6 with lanes 8-12).
Quantification of these data reveals an increase of approximately $20 \%-25 \%$ in pre-miR-422a in reactions containing MutL $\alpha$ (Figure 5B). Similar results were obtained in the processing reactions with pri-miR-30a (Figure $5 \mathrm{C}$ ) and pri-miR-16-1 (Figure 5D) in the presence of MutL $\alpha$.

To determine the MutL $\alpha$ effect on miRNA processing in vivo, we transfected a plasmid carrying either a pri-miRNA gene (pri-miR-422a or pri-miR-16-1) or a scrambled sequence into MLH1-deficient HCT116 cells with or without MLH1 expression, and the conversion to pre-miRNA and mature miRNA from the exogenous primiRNA was detected by northern blotting analysis using a DNA oligomer complementary to the mature miRNA as a probe. It was found that enhanced production of premiRNA and mature miRNA was observed in HCT116 cells transfected with $M L H 1$ for both miR-422a (Figure 5E) and miR-16-1 (Figure 5F). Taken together, our results shown here suggest that MutL $\alpha$ stimulates Drosha/ DGCR8-mediated processing of pri-miRNA to premiRNA in vitro and in vivo.

MutL $\alpha$ ATPase and pri-miRNA binding activities are required for stimulation of miRNA processing

MutL $\alpha$ has an intrinsic ATPase activity that is required
A

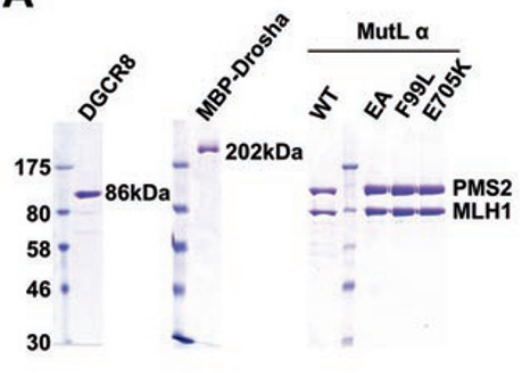

D

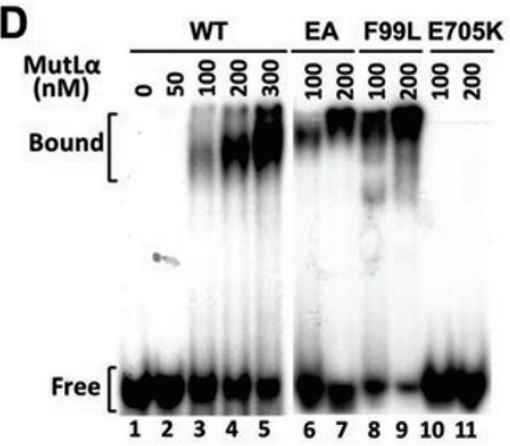

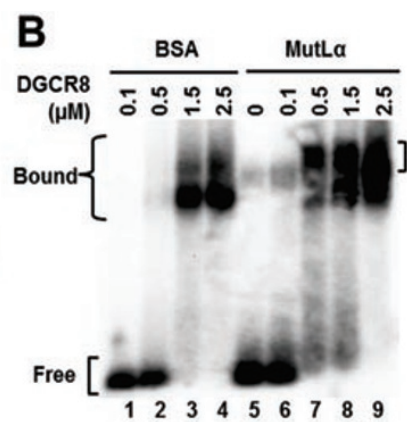

E

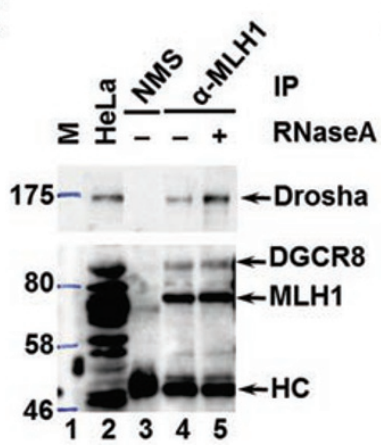

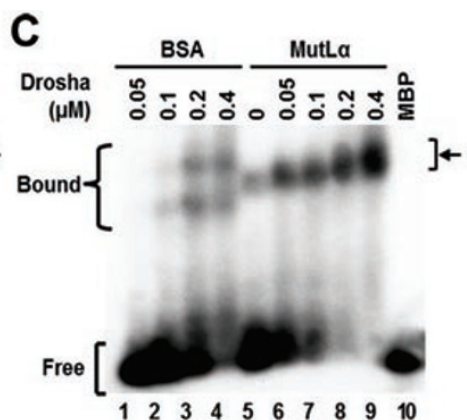

$\mathbf{F}$

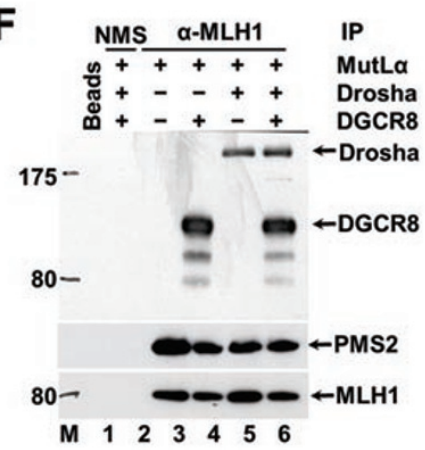

Figure 4 MutL $\alpha$ interacts with Microprocessor and pri-miR-422a. (A) Purified recombinant proteins used in this study. (B, C) Effect of MutL $\alpha$ on pri-miR-422a interactions with DGCR8 (B) and Drosha (C). (D) Binding of pri-miR-422a by wild-type (WT) and mutant MutL $\alpha$ proteins. (E, F) Co-immunoprecipitation (Co-IP) of MLH1 and Microprocessor in HeLa whole-cell extracts $(E)$ and purified proteins (F). Co-IP reactions were performed using an MLH1 antibody and individual proteins were detected using their corresponding antibodies, as indicated. NMS, non-immune mouse serum; HC, IgG heavy chain. 

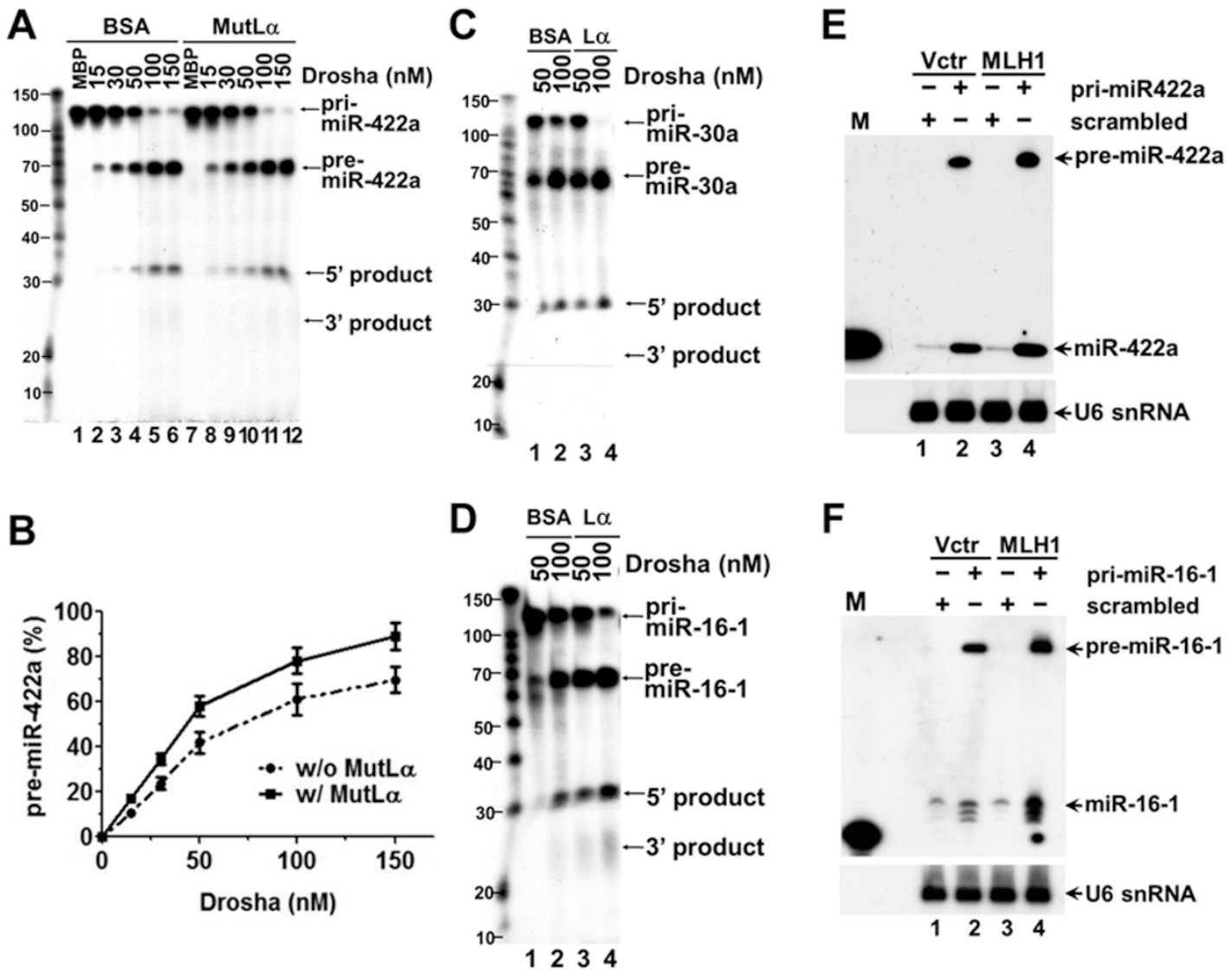

Figure 5 MutL $\alpha$ promotes pri-miR-422a processing. (A, B) In-vitro reconstitution of miRNA processing. Reconstitution of miRNA processing was performed in reactions containing pri-miR-422a, Drosha and DGCR8 in the presence or absence of MutL $\alpha$, as indicated. Three independent experiments were performed. Data were quantified, presented as mean \pm SD, and plotted in (B). (C, D) Stimulation of Drosha/DGCR8-mediated processing of pre-miR-30a and pre-miR-16-1 by MutL $\alpha$, respectively. (E, F) MutL $\alpha$ stimulates the processing of miR-422a and miR-16-1 in vivo, respectively. HCT116 cells with or without MLH1 expression were transfected with plasmids carrying the indicated pri-miRNAs, and RNAs were isolated $48 \mathrm{~h}$ after transfection and analyzed for conversion of the exogenous pri-miRNAs to their corresponding pre- and mature miRNAs using northern blotting analysis as described [49]. U6 snRNA was used as a loading control. M, a 23-oligonucleotide size marker; L $\alpha$, MutL $\alpha$; Vctr, vector alone.

for its function in MMR [33, 34]. We therefore examined whether ATP hydrolysis by MutL $\alpha$ is essential for its ability to promote pri-miRNA processing. As shown in Figure 6A, MutL $\alpha$ stimulates pri-miRNA processing in the presence of ATP (compare lanes 4 and 5 with lane 2). However, the stimulation was not observed when ATP was replaced with a non-hydrolyzable ATP analog, AMPPNP, in the reaction (Figure 6A, lanes 6 and 7). Instead, an uncharacterized slow-migrating RNA species accumulated in the presence of AMP-PNP (Figure 6A, asterisk). These observations indicate that ATP hydrolysis by MutL $\alpha$ is essential for its ability to stimulate miRNA processing.

This idea was further examined using MMR-deficient MutL $\alpha$ proteins MutL $\alpha$ EA, MutL $\alpha$ F99L and MutL $\alpha E 705 \mathrm{~K}$
$[29,34,35]$. Our results show that MutL $\alpha$ EA and MutL $\alpha$ F99L are proficient, but MutL $\alpha$ E705K is deficient in pri-miRNA binding (Figure 4D). In addition, MutL $\alpha E A$ hydrolyzes ATP, but generates different products than the WT MutL $\alpha$ (Figure 6B, compare lanes 1 and 2 with lanes 3 and 4), while MutL $\alpha$ F99L (Figure 6B, lanes 5, 6) and MutLaE705K (Figure 6B, lanes 7,8) exhibit nearly normal ATPase activity. Figure 6C shows reconstituted miRNA processing reactions in the presence or absence of these MutL $\alpha$ mutants. MutL $\alpha$ F99L stimulates the reaction as efficiently as WT MutL $\alpha$ (Figure 6C, compare lanes 2-3 with 9-10). However, MutL $\alpha$ EA and MutL $\alpha E 705 \mathrm{~K}$ have lost the ability to stimulate this reaction; instead, two novel species accumulate, one migrating immediately below the substrate and the other below 
the normal reaction product (Figure 6C, asterisks). These species, similar to those generated in reactions containing AMP-PNP (Figure 6A, lanes 6 and 7), may be nonspecific reaction products produced by aborted processing reactions [36]. These observations suggest that MutL $\alpha$ must retain both pri-miRNA binding and normal ATPase activities in order to stimulate miRNA processing by Microprocessor.

MutLa compensates for the loss of basal segments of primiRNA

Previous studies indicate that the single-stranded RNA segments at the $3^{\prime}$ - and $5^{\prime}$-termini of pri-miRNA, also known as basal segments, are essential for pri-miRNA processing by Drosha and DGCR8 [36, 37]. Our studies confirm that the Drosha-DGCR8 Microprocessor converted pri-miR-16-1 to pre-miR-16-1 with high efficiency (Figure 6D, lanes 1-2), but the same substrate lacking basal segments (i.e., pri-miR-16-1 $\Delta \mathrm{BS}$ ) was poorly processed by the Microprocessor complex, as judged by the fact that a very small amount pre-miR-16-1 and several other fast-migrating RNA species (likely nonspecific reaction products) were produced (Figure 6D, lane 6). However, MutL $\alpha$ caused a dose-dependent increase in the specific product and decrease in the non-specific reaction products (Figure 6D, lanes 7-9). These findings suggest that pri-miRNA basal segments are essential for miRNA processing by Drosha/DGCR8 in the absence of MutL $\alpha$, but are not required when MutL $\alpha$ is present. In other words, MutL $\alpha$ restores normal reaction efficiency and specificity when the pri-miRNA substrate lacks basal segments. EMSA assays confirmed that MutL $\alpha$ binds efficiently to pri-miR-16-1 $\Delta \mathrm{BS}$ (data not shown). We also examined the effect of MutL $\alpha$ on the processing of primiRNAs with a nicked terminal loop, as whether or not the terminal loop is required for efficient processing of pri-miRNAs is rather controversial [36-39]. However, our results reveal that in the purified system, Microprocessor by itself can efficiently process pri-miR-16-1 containing a nicked terminal loop, although MutL $\alpha$ can stimulate the reaction (data not shown). Collectively, we demonstrate that MutL $\alpha$ promotes a Microprocessor-mediated processing of miR-422a, as well as several other miRNAs tested, including those lacking basal segments.

\section{Discussion}

In this study, we made the following interesting observations: (1) discovery of a novel MutL $\alpha$ function in stimulating miRNA processing; (2) identification of a
A

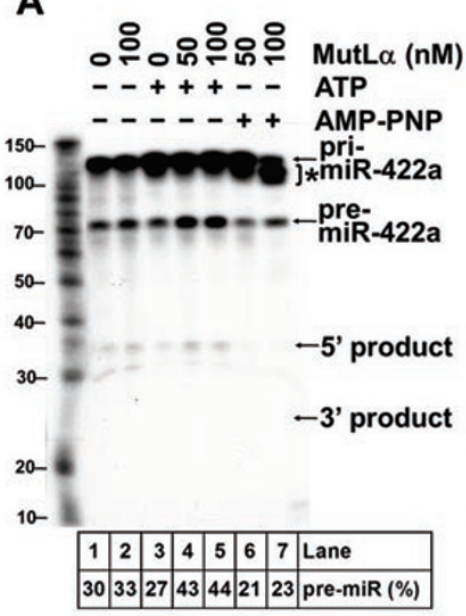

B

B

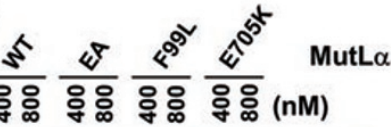

D

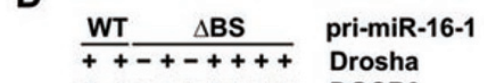

$++\overline{++++++}$ Drosha

$++\div++++$ DGCR8

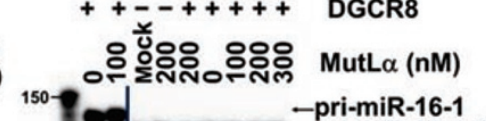

$\begin{array}{llll}12 & 34 & 56 & 78\end{array}$

C

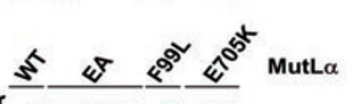

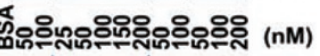

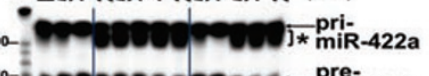

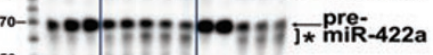

5' product

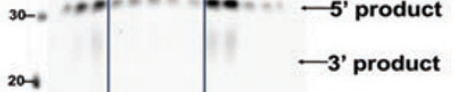

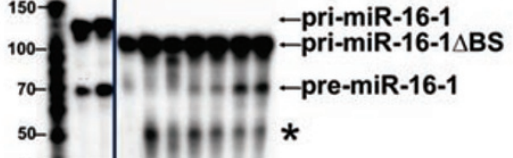

.

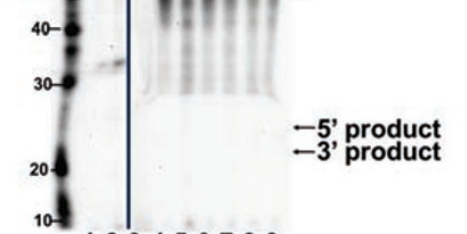

123456789

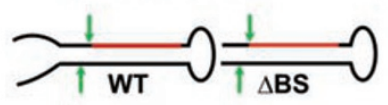

Figure 6 Processing enhancement by MutL $\alpha$ requires integrity of the MutL $\alpha$ ATPase and pri-miRNA binding activity. (A) Effect of ATP on miRNA processing stimulation by MutL $\alpha$. In-vitro processing of pri-miR-422a was carried out in the presence of 2 mM ATP or AMP-PNP, as indicated. pri-miR-422a and the resulting pre-miR-422a and 3'-, 5'-products are indicated with arrows. (B) ATPase assay of wild-type (WT) and mutant MutL $\alpha$ proteins. The indicated proteins were incubated with $330 \mathrm{mM}$ $\left(\gamma-{ }^{32} \mathrm{P}\right)$-ATP in reactions containing $50 \mathrm{mM}$ Hepes. $\mathrm{KOH}, \mathrm{pH} 7.6$ and $10 \mathrm{mM} \mathrm{MgCl}$, and the hydrolysis products were analyzed in a $20 \%$ polyacrylamide gel. (C) In-vitro processing of pri-miR-422a using WT MutL $\alpha$, MutLaEA (EA), MutL $\alpha$ F99L (F99L) or MutL $\alpha$ E705K (E705K). (D) In-vitro processing of WT pri-miR-16-1 and flanking basal segment deleted pri-miR-16-1 $\Delta \mathrm{BS}(\Delta \mathrm{BS})$. *Non-specific reaction products. 


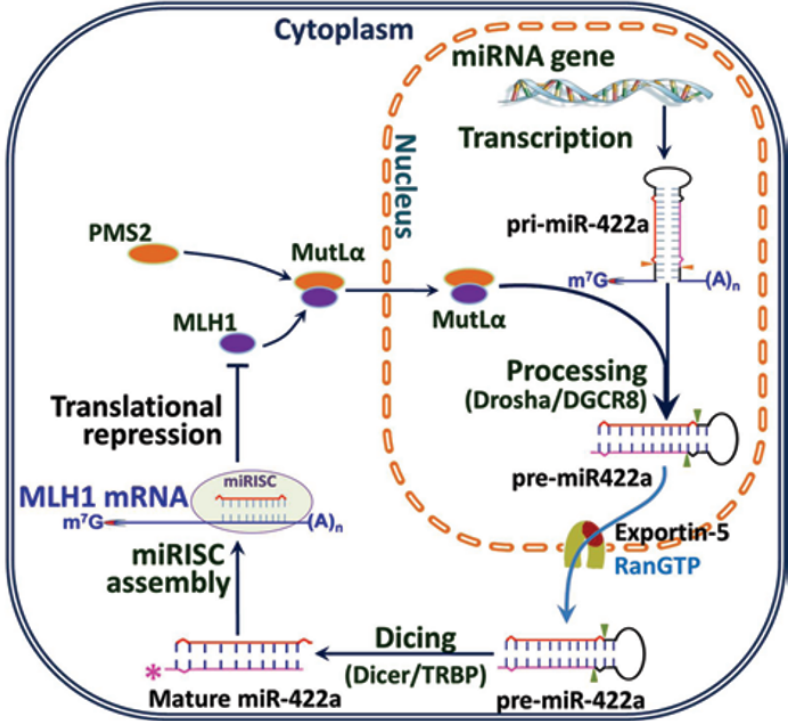

Figure 7 Model for reciprocal feedback regulation between MLH1 and miR-422a. MLH1 dimerizes with PMS2 to form MutL $\alpha$ in the cytoplasm, and the MutLa heterodimer then translocates into the nucleus to participate in genome maintenance via the MMR system. When MutL $\alpha$ reaches to a level that is toxic to cells, it begins to stimulate the conversion of pri-miR-422a into pre-miR-422a, leading to increasing production of pre-miR-422a and subsequently mature miR-422a. The resulting high level of mature miR-422a interacts with MLH1 mRNA by pairing with a putative miR-422a interaction sequence in the MLH1 3'-UTR, which inhibits MLH1 translation.

regulatory feedback between miR-422a and MLH1; (3) reconstitution of the miRNA processing reaction in a defined system.

We initially observed a positive correlation between miR-422a processing and MLH1 expression (Figures 2 and 3), and later showed that the correlation is due to direct stimulation of the miRNA processing by MutL $\alpha$ (Figure 5). Since MutL $\alpha$ interacts with pri-miRNAs and the Microprocessor complex (Figure 4), and also promotes the processing of several other miRNAs tested (Figure 5), we believe that the newly identified MutL $\alpha$ activity in miRNA biogenesis likely applies to many other miRNAs, if not all. This idea is consistent with the fact that MLH1 dictates many miRNA expression patterns in colorectal tumors $[27,28]$.

The mechanism by which MutL $\alpha$ stimulates miRNA processing is not clear. Nonetheless, the data shown in this study indicate that the stimulation reaction requires MutL $\alpha$ ATPase activity and two novel activities identified in this study (Figure 6), i.e., pri-miRNA binding and interactions with Drosha and DGCR8. Because ATP hydrolysis by MutL leads to conformational changes of the protein, which modulate interactions between MutL and other MMR components [40, 41], it is possible that ATP hydrolysis by MutL $\alpha$ during the pri-miRNA processing reaction promotes a required conformational change of MutL $\alpha$ and/or its binding partners in the context of this reaction. This possibility is consistent with the observation that MutL $\alpha \mathrm{EA}$, which is defective in normal ATPase activity (Figure 6B), does not stimulate, but inhibits, miRNA processing (Figure 6C). Similarly, the MutL $\alpha$ mutant that lacks pri-miRNA-binding activity also fails to stimulate miRNA processing (see MutL $\alpha$ E705K in Figure 6C). The most important aspect of the MutL $\alpha$ mediated stimulation of miRNA processing may be the interaction between MutL $\alpha$ and Microprocessor, which may allow MutL $\alpha$ to independently enhance the affinity of Drosha and/or DGCR8 for its pri-miRNA substrate (Figure 4B and 4C). However, the mechanism underlying these interactions remains to be investigated.

It is worth mentioning that MutL $\alpha$ has recently been shown to possess a latent endonuclease activity, but the E705K substitution in the PMS2 subunit of MutL $\alpha$ abolishes the endonuclease activity [35]. Therefore, it is possible that the observed stimulation of miRNA processing by MutL $\alpha$ may be attributed to the protein endonuclease activity. However, based on the fact that miRNA processing requires site-specific cleavages and that MutL $\alpha$ is a non-specific nuclease, whose activation requires replication factor $\mathrm{C}$ and proliferating cellular nuclear antigen [35], which were not present in our reactions, we believe that the MutL $\alpha$ endonuclease activity is unlikely involved in stimulating miRNA processing.

A second way by which MutL $\alpha$ enhances pre-miRNA production is to ensure the conversion of pri-miRNAs that lack basal segments to pre-miRNAs. Previous studies have shown that miRNA basal segments are required for Drosha/DGCR8-mediated processing of pri-miRNA to pre-miRNA [36]. We demonstrate here that the presence of MutL $\alpha$ in the miRNA processing reaction is sufficient to compensate for the loss of miRNA basal segments. However, further studies are required to understand the molecular basis of this reaction.

While MutL $\alpha$ (MLH1-PMS2) facilitates miRNA processing, we also show that expression of MLH1 is regulated by at least miR-422a. MutL $\alpha$ is known to be involved in all known genome-maintenance functions of the MMR system, including correction of DNA biosynthetic errors, suppression of homeologous recombination and DNA damage signaling [20-23, 42]. In addition, MLH1 plays a role in DNA interstand crosslink repair [43] and spermatogenesis $[44,45]$. Interestingly, overexpression of MLH1 does not enhance genome stability, but promotes hypermutability $[25,26]$. Because MLH1 is a 
critical component of multiple protein complexes and has multiple important cellular functions, the cellular level of MLH1 is likely to be subject to tight regulation. The reciprocal feedback regulation between MLH1 and miR422a may represent one of the mechanisms by which cells regulate MLH1 expression. A model describing this feedback regulation is depicted in Figure 7.

In this model, MLH1 and miR-422a reciprocally regulate each other's cellular level. This relationship has several implications: First, a high level of MLH1 stimulates miR-422a processing. MLH1 dimerizes with PMS2 in the cytoplasm to form MutL $\alpha$, and the MutL $\alpha$ heterodimer then translocates into the nucleus [46], where it participates in genome maintenance via the MMR system. However, as the concentration of MutL $\alpha$ increases, it stimulates conversion of pri-miR-422a into pre-miR$422 \mathrm{a}$, leading to increased level of pre-miR-422a. PremiR-422a is transported into the cytoplasm and further processed into mature miR-422a. Second, as the concentration of mature miR-422a increases, it inhibits MLH1 expression. This inhibition is through pairing of the mature miRNA with the putative miR-422a-binding sequence in the MLH1 3'-UTR, suppressing MLH1 translation (this study). We believe that this mechanism is critical for precise regulation of the cellular level of MLH1. An important feature of this model is that high or low thresholds of MLH1 concentration trigger a cellular response. When MLH1 protein falls below a critical level, all MLH1 molecules are recruited into genome-maintenance activities, and MLH1 is not available to stimulate Drosha/DGCR8-mediated miRNA processing reactions. Thus, the rate of pri-miR-422a processing decreases, leading to a reduced level of mature miR-422a. In contrast, when the MLH1 concentration increases above the level required for genome-maintenance, it becomes toxic and cell viability declines [24-26]. In this situation, excess MutL $\alpha$ stimulates processing of pri-miR-422a, and the resulting mature miR-422a suppresses MLH1 translation, thus preventing toxic effects of MLH1 overexpression. However, whether or not other miRNAs participate in a similar feedback regulation with MLH1 awaits further investigations.

\section{Materials and Methods}

\section{DNA constructs}

A synthetic DNA oligonucleotide was prepared using the DNA sequence of the putative miR-422aRE located between +18 and +50 of the MLH1 3'-UTR. Three tandem copies of this olignocleotide $(3 \times$ miR422aRE) were subcloned into the HindIII and SpeI sites of pMIR-REPORT Luciferase (Ambion, Inc), to produce pMIR-Luc-3 $\times$ miR422aRE. pMIR-MLH1-3×miR422aRE was constructed from pMIR-Luc-3×miR422aRE by replacing the Lu- ciferase gene with the $M L H 1$ cDNA.

The MLH1 cDNA, with or without the MLH1 3'-UTR or $3 \times$ miR422aRE, was subcloned into pcDNA3.1/His ABC (Invitrogen Corp.). The stop codon TAA was substituted for the ATG start codon, as indicated in Figure 3B, using QuikChange Site-Directed Mutagenesis Kit (Stratagene). Cloning strategies for all reporter and MLH1 expression constructs used in this study are shown in Figures 1D, 3A and 3B. Primers used to detect pri- and pre-miR422a are shown in Figure 2A, and their specific sequences are provided in Supplementary information, Tables S1-S4. The sequences of all plasmids were confirmed by DNA sequencing.

\section{Cell culture and transfection}

HeLa, HEK293, 293T and HCT116 cells were cultured in media recommended by ATCC supplemented with $10 \%$ fetal bovine serum. Transfections were carried out using 25-200 pmol of the indicated pre-miR-422a or anti-miR-422a, with or without $2 \mu \mathrm{g}$ plasmid DNA, as indicated, and FuGENE ${ }^{\circledR}$ HD Transfection Reagent (Roche Diagnostics GmbH, Germany). The medium was switched $48 \mathrm{~h}$ after transfection. Total RNA and cellular protein was isolated from transfected cells. Alternatively, luciferase activity was measured in transfected cells.

\section{Total RNA extraction, RT-PCR and $q R T-P C R$}

Unless mentioned otherwise, total RNAs were used to determine mRNA levels of $M L H 1$ and $\beta$-actin (internal control), and RNAs isolated from cytoplasm and nuclei were employed to determine the levels of pre- and pri-miRNAs, respectively, using Qiagen RNeasy Mini Kit (Qiagen). RNAs $(1 \mu \mathrm{g})$ were treated with RQ1 DNase (Promega Corp.) and used for first-strand cDNA synthesis in a $100-\mu 1$ reaction containing TaqMan Reverse Transcription Reagents (Applied Biosystems). cDNA from each sample was diluted 1:10 in qRT-PCR buffer. qRT-PCR reactions were performed using a 7300 Real-Time PCR System (Applied Biosystems), with SYBR ${ }^{\circledR}$ Green PCR Master Mix $(2 \times)$ Kit (Applied Biosystems). All reactions were carried out in quadruplicate with reference dye normalization, and the median $\mathrm{Ct}$ value was used for statistical analysis. qRT-PCR was carried out in triplicate. All values were normalized to $\beta$-actin. Primer sequences used for quantitative PCR studies are listed in Supplementary information, Tables S1-S4.

\section{RNA interference}

Human MLH1 siRNA duplex was from Santa Cruz Biotechnology, Inc. HeLa cells were transfected with 100 pmol MLH1 siRNA duplex in six-well plates with FuGENE ${ }^{\circledR}$ HD Transfection Reagent (Roche Diagnostics GmbH, Germany). After 48 h, cells were harvested and processed for RNA quantification by RT- and/or qRTPCR or protein quantification by western blotting.

\section{Protein expression and purification}

Drosha cDNA was subcloned into pFastBac-1 (Invitrogen) with an MBP-tag at N-terminus, and a tandem Flag- and $6 \times$ Histag at $\mathrm{C}$-terminus. The resulting expression construct, $\mathrm{pFB}$-MBPDrosha-FH, was used to overexpress MBP-Drosha in High Five ${ }^{\mathrm{TM}}$ insect cells. Drosha was purified from the insect cells through affinity column chromatography, initially with a nickel HisTrap ${ }^{\mathrm{TM}}$ HP column (GE Healthcare) and later with an Amylose Resin column (New England Biolabs, Inc.), followed by dialysis against the 
PBE100 Buffer (25 mM HEPES-NaOH, pH 7.8, $100 \mathrm{mM} \mathrm{NaCl}$, $1 \mathrm{mM}$ DTT, $0.1 \mathrm{mM}$ EDTA and $10 \%$ glycerol) for $4 \mathrm{~h}$ at $4{ }^{\circ} \mathrm{C}$. Aliquots of MBP-Drosha were added $1 \mathrm{mg} / \mathrm{ml} \mathrm{BSA}$, and frozen in liquid nitrogen and stored at $-80{ }^{\circ} \mathrm{C}$ for use.

Similarly, DGCR8 cDNA was cloned into the EcoRI and HindIII sites of pFastBac-HTc (Invitrogen), and the recombinant protein containing an N-terminal tandem $6 \times$ His- and a Flag-tag C-terminus was expressed in High Five ${ }^{\mathrm{TM}}$ insect cells and purified using a HisTrap ${ }^{\mathrm{TM}}$ HP nickel column (GE Healthcare). The peak fractions containing DGCR8 were dialyzed against the PBE100 buffer for $4 \mathrm{~h}$ at $4{ }^{\circ} \mathrm{C}$, and further purified using an ANTI-FLAG M2 affinity gel (Sigma-Aldrich). Briefly, the dialyzed DGCR8 was incubated with the affinity gel at $4{ }^{\circ} \mathrm{C}$ overnight, washed with fiveresin volumes of the PBE100 buffer, and eluted with $3 \times$ FLAG peptide $(0.3 \mathrm{mg} / \mathrm{ml}$ in PBE100). Aliquots of purified DGCR8 were adjusted to $1 \mathrm{mg} / \mathrm{ml} \mathrm{BSA}$, frozen in liquid nitrogen and stored at $-80^{\circ} \mathrm{C}$ for future use.

Constructs of expression of MutL $\alpha$ and MutL $\alpha$ EA were gifts from Michael Liskay (Oregon Health Sciences University), and constructs for MutL $\alpha$ F99L and MutL $\alpha$ E705K were generated using the QuikChange ${ }^{\circledR}$ Site-Directed Mutagenesis Kit (Stratagene). All MutL heterodimers were expressed in pFastBac Dual vector and purified as described $[34,47]$. The purity of all recombinant proteins was assessed by SDS-PAGE and protein concentrations were determined by Bradford assay.

\section{Preparation of miRNA processing substrates}

pri-miR-422a, pri-miR-145, pri-miR-30a, pri-miR-16-1, primiR16-sTL1 and pri-miR-16-1 $\triangle B S$ were designed, generated and purified as described previously [36]. PCR products contained the T7-promoter sequence and two tandem G's right before primiRNA sequences, and were amplified from a normal human whole-blood genomic DNA sample. The T7-primer sequences are available upon request. The $\mathrm{T} 7-\mathrm{G}_{2}$-pri-miRNA PCR products were used directly as templates for in-vitro transcription of pri-miRNA substrates with MEGAshortscript ${ }^{\mathrm{TM}}$ Kit (Ambion, Inc.). The primiRNA transcripts were radiolabeled internally by incubating with $\left[\alpha-{ }^{32} \mathrm{P}\right]$-ATP in the transcription reactions according to the manufacturer's instruction.

\section{Electrophoretic mobility shift assay}

EMSA was performed in $20-\mu 1$ reactions containing buffer PBE100, Drosha, DGCR8, 1.0 U SUPERase $\cdot$ In $^{\mathrm{TM}}$ RNase Inhibitor (Ambion, Inc.), and 200 pmol ${ }^{32} \mathrm{P}$-labeled pri-miR-422a in the presence or absence of MutL $\alpha$ or MutL $\alpha$ mutants, as indicated. Unlabeled tRNAs (10× excess) were included as non-specific competitor. Reactions were incubated for $20 \mathrm{~min}$ on ice, followed by $5 \mathrm{~min}$ at room temperature. Reaction products were loaded onto $4 \%$ non-denaturing polyacrylamide gels in $0.5 \times \mathrm{TBE}$. Gels were run at $150 \mathrm{~V}$ for $3 \mathrm{~h}$ at $4{ }^{\circ} \mathrm{C}$, and then quantified by phosphorimaging using a KODAK Molecular Imager System.

\section{Immunoprecipitation analysis}

Co-immunoprecipitation of Drosha, DGCR8 and MutL $\alpha$ was conducted by incubating either HeLa whole-cell extracts $(500 \mu \mathrm{g})$ or purified proteins (100 nM Drosha, $200 \mathrm{nM}$ DGCR8 and 100 $\mathrm{nM}$ MutL $\alpha$ ) with an MLH1 antibody (BD Pharmingen, 554073) under the conditions for in-vitro pri-miRNA processing (see below). MBP or BSA was used as negative controls for the purified system. Immunoprecipitates were analyzed by $8 \%$ SDS-PAGE and simultaneously immunoblotted with rabbit antibodies against DGCR8 (ProteinTech Group, Inc), Drosha (Bethyl Laboratories, Inc) and MBP (ProteinTech Group, Inc).

\section{Reconstitution of pri-miRNA processing}

The conditions for in-vitro pri-miRNA processing were essentially the same as described $[6,48]$, except that purified proteins were used in this study. Unless indicated otherwise, pri-miRNA processing assays were performed in $30-\mu 1$ reactions, containing $100 \mathrm{nM}$ Drosha, $200 \mathrm{nM}$ DGCR8, $100 \mathrm{nM}$ MutL $\alpha$ (or mutant variants indicated), $6.4 \mathrm{mM} \mathrm{MgCl}_{2}, 2 \mathrm{mM}$ ATP, 1.0 U SUPERase•In RNase Inhibitor and 200 pmol ${ }^{32} \mathrm{P}$-labeled pri-miRNA substrate in buffer PBE100. BSA was included in reactions lacking MutL $\alpha$, and MBP was included in reactions lacking Drosha. The reaction mixture was incubated at $37{ }^{\circ} \mathrm{C}$ for $90 \mathrm{~min}$ (or as indicated) and terminated by phenol extraction. The RNA products were analyzed by $12.5 \%$ denaturing PAGE in $0.5 \times$ TBE. Gels were run at $350 \mathrm{~V}$ until bromophenol blue reached the bottom of the gel (about $2 \mathrm{~h}$ ). Radiolabeled species were visualized by autoradiography and/or PhosphorImager, and quantified using KODAK Molecular Imager Systems (KODAK MI) version 5.0 (EASTMAN KODAK).

\section{Statistical analysis}

The data shown in this study presented an average of at least three independent experiments, each performed in triplicate. All statistical assays, Student's $t$-test and one-way ANOVA with Tukey's multiple comparison, were performed using GraphPad Prism 5.0 (GraphPad Software, Inc.). Data were considered statistically significant if $P$-values were less than 0.05 or 0.001 , as indicated.

\section{Acknowledgments}

We are grateful to VN Kim (Seoul National University, Korea) for providing human Flag-Drosha and Flag-DGCR8 cDNAs, to Ramin Shiekhattar (The Wistar Institute, USA) for pCMV-DGCR8 clone, to Feng Guo (University of California, Los Angeles, USA) for $\mathrm{pFastBac}-6 \times$ His-Drosha vector, to Michael Liskay (Oregon Health \& Science University, USA) for baculovirus and plasmid constructs expressing WT and mutant MutL $\alpha$ proteins, and to H-S Yang (University of Kentucky, USA) for technical assistance on the luciferase analysis. This work was supported by National Institutes of Health Grants CA104333 (to LG), GM072756 and CA115942 (to GML). GML is the James-Gardner Chair in Cancer Research.

\section{References}

1 Bartel DP. MicroRNAs: target recognition and regulatory functions. Cell 2009; 136:215-233.

2 Fabian MR, Sonenberg N, Filipowicz W. Regulation of mRNA translation and stability by microRNAs. Annu Rev Biochem 2010; 79:351-379.

3 Kim VN, Han J, Siomi MC. Biogenesis of small RNAs in animals. Nat Rev Mol Cell Biol 2009; 10:126-139.

4 Denli AM, Tops BB, Plasterk RH, Ketting RF, Hannon GJ. Processing of primary microRNAs by the Microprocessor complex. Nature 2004; 432:231-235. 
5 Gregory RI, Yan KP, Amuthan G, et al. The Microprocessor complex mediates the genesis of microRNAs. Nature 2004; 432:235-240.

6 Han J, Lee Y, Yeom KH, Kim YK, Jin H, Kim VN. The Drosha-DGCR8 complex in primary microRNA processing. Genes Dev 2004; 18:3016-3027.

7 Landthaler M, Yalcin A, Tuschl T. The human DiGeorge syndrome critical region gene 8 and its D. melanogaster homolog are required for miRNA biogenesis. Curr Biol 2004; 14:21622167.

8 Suzuki HI, Yamagata K, Sugimoto K, Iwamoto T, Kato S, Miyazono K. Modulation of microRNA processing by $\mathrm{p} 53$. Nature 2009; 460:529-533.

9 Davis BN, Hilyard AC, Nguyen PH, Lagna G, Hata A. Smad proteins bind a conserved RNA sequence to promote microRNA maturation by Drosha. Mol Cell 2010; 39:373-384.

10 Davis BN, Hilyard AC, Lagna G, Hata A. SMAD proteins control DROSHA-mediated microRNA maturation. Nature 2008; 454:56-61.

11 Zhang X, Wan G, Berger FG, He X, Lu X. The ATM kinase induces microRNA biogenesis in the DNA damage response. Mol Cell 2011; 41:371-383.

12 Mao G, Pan X, Gu L. Evidence that a mutation in the MLH1 3 '-untranslated region confers a mutator phenotype and mismatch repair deficiency in patients with relapsed leukemia. $J$ Biol Chem 2008; 283:3211-3216.

13 Valeri N, Gasparini P, Fabbri M, et al. Modulation of mismatch repair and genomic stability by miR-155. Proc Natl Acad Sci USA 2010; 107:6982-6987.

14 Valeri N, Gasparini P, Braconi C, et al. MicroRNA-21 induces resistance to 5-fluorouracil by down-regulating human DNA MutS homolog 2 (hMSH2). Proc Natl Acad Sci USA 2010; 107:21098-21103.

15 Fishel R, Kolodner RD. Identification of mismatch repair genes and their role in the development of cancer. Curr Opin Genet Dev 1995; 5:382-395.

16 Kinzler KW, Vogelstein B. Lessons from hereditary colorectal cancer. Cell 1996; 87:159-170.

17 Kolodner RD, Alani E. Mismatch repair and cancer susceptibility. Curr Opin Biotechnol 1994; 5:585-594.

18 Lynch HT, de la Chapelle A. Genetic susceptibility to nonpolyposis colorectal cancer. J Med Genet 1999; 36:801-818.

19 Modrich P, Lahue R. Mismatch repair in replication fidelity, genetic recombination, and cancer biology. Annu Rev Biochem 1996; 65:101-133.

20 Hsieh P, Yamane K. DNA mismatch repair: molecular mechanism, cancer, and ageing. Mech Ageing Dev 2008; 129:391407.

21 Jiricny J. The multifaceted mismatch-repair system. Nat Rev Mol Cell Biol 2006; 7:335-346.

22 Kunkel TA, Erie DA. DNA mismatch repair. Annu Rev Biochem 2005; 74:681-710.

23 Li GM. Mechanisms and functions of DNA mismatch repair. Cell Res 2008; 18:85-98.

24 Zhang H, Richards B, Wilson T, et al. Apoptosis induced by overexpression of $h M S H 2$ or hMLH1. Cancer Res 1999; 59:3021-3027.

25 Shcherbakova PV, Hall MC, Lewis MS, et al. Inactivation of DNA mismatch repair by increased expression of yeast
MLH1. Mol Cell Biol 2001; 21:940-951.

26 Shcherbakova PV, Kunkel TA. Mutator phenotypes conferred by $M L H 1$ overexpression and by heterozygosity for $m l h 1 \mathrm{mu}-$ tations. Mol Cell Biol 1999; 19:3177-3183.

27 Oberg AL, French AJ, Sarver AL, et al. miRNA expression in colon polyps provides evidence for a multihit model of colon cancer. PLoS One 2011; 6:e20465.

28 Sarver AL, French AJ, Borralho PM, et al. Human colon cancer profiles show differential microRNA expression depending on mismatch repair status and are characteristic of undifferentiated proliferative states. BMC Cancer 2009; 9:401.

29 Mao G, Yuan F, Absher K, et al. Preferential loss of mismatch repair function in refractory and relapsed acute myeloid leukemia: potential contribution to AML progression. Cell Res 2008; 18:281-289.

30 Rusinov V, Baev V, Minkov IN, Tabler M. MicroInspector: a web tool for detection of miRNA binding sites in an RNA sequence. Nucleic Acids Res 2005; 33:W696-W700.

31 Cejka P, Stojic L, Mojas N, et al. Methylation-induced G(2)/ $\mathrm{M}$ arrest requires a full complement of the mismatch repair protein hMLH1. EMBO J 2003; 22:2245-2254.

32 Lee Y, Jeon K, Lee JT, Kim S, Kim VN. MicroRNA maturation: stepwise processing and subcellular localization. EMBO $J$ 2002; 21:4663-4670.

33 Raschle M, Dufner P, Marra G, Jiricny J. Mutations within the hMLH1 and hPMS2 subunits of the human MutLalpha mismatch repair factor affect its ATPase activity, but not its ability to interact with hMutSalpha. J Biol Chem 2002; 277:21810-21820.

34 Tomer G, Buermeyer AB, Nguyen MM, Liskay RM. Contribution of human mlh1 and pms2 ATPase activities to DNA mismatch repair. J Biol Chem 2002; 277:21801-21809.

35 Kadyrov FA, Dzantiev L, Constantin N, Modrich P. Endonucleolytic function of MutLalpha in human mismatch repair. Cell 2006; 126:297-308.

36 Han J, Lee Y, Yeom KH, et al. Molecular basis for the recognition of primary microRNAs by the Drosha-DGCR8 complex. Cell 2006; 125:887-901.

37 Zeng Y, Cullen BR. Efficient processing of primary microRNA hairpins by Drosha requires flanking nonstructured RNA sequences. J Biol Chem 2005; 280:27595-27603.

38 Zeng Y, Yi R, Cullen BR. Recognition and cleavage of primary microRNA precursors by the nuclear processing enzyme Drosha. EMBO J 2005; 24:138-148.

39 Zhang X, Zeng Y. The terminal loop region controls microRNA processing by Drosha and Dicer. Nucleic Acids Res 2010; 38:7689-7697.

40 Ban C, Junop M, Yang W. Transformation of MutL by ATP binding and hydrolysis: a switch in DNA mismatch repair. Cell 1999; 97:85-97.

41 Ban C, Yang W. Crystal structure and ATPase activity of MutL: implications for DNA repair and mutagenesis. Cell 1998; 95:541-552.

42 Iyer RR, Pluciennik A, Burdett V, Modrich PL. DNA mismatch repair: functions and mechanisms. Chem Rev 2006; 106:302-323.

$43 \mathrm{Wu}$ Q, Vasquez KM. Human MLH1 protein participates in genomic damage checkpoint signaling in response to DNA interstrand crosslinks, while MSH2 functions in DNA repair. 
PLoS Genet 2008; 4:e1000189.

44 Baker SM, Plug AW, Prolla TA, et al. Involvement of mouse Mlh1 in DNA mismatch repair and meiotic crossing over. Nat Genet 1996; 13:336-342.

45 Edelmann W, Cohen PE, Kane M, et al. Meiotic pachytene arrest in MLH1-deficient mice. Cell 1996; 85:1125-1134.

46 Wu X, Platt JL, Cascalho M. Dimerization of MLH1 and PMS2 limits nuclear localization of MutLalpha. Mol Cell Biol 2003; 23:3320-3328.
47 Zhang Y, Yuan F, Presnell SR, et al. Reconstitution of 5'-directed human mismatch repair in a purified system. Cell 2005; 122:693-705.

48 Faller M, Matsunaga M, Yin S, Loo JA, Guo F. Heme is involved in microRNA processing. Nat Struct Mol Biol 2007; 14:23-29.

49 Koscianska E, Starega-Roslan J, Czubala K, Krzyzosiak WJ. High-resolution northern blot for a reliable analysis of microRNAs and their precursors. Sci World J 2011; 11:102-117.

(Supplementary information is linked to the online version of the paper on the Cell Research website.) 